\title{
SARCOMA OF THE PULMONARY ARTERY
}

\author{
BY \\ R. H. ELPHINSTONE AND R. G. SPECTOR* \\ From Edmonton Chest Clinic and Chase Farm Hospital, Enfield, Middlesex
}

(RECEIVED FOR PUBLICATION JUNE 29, 1959)

Primary sarcomata of the great vessels are so rare that Jores, in 1924, dismissed all cases described up to that time, for lack of evidence of their nature. Eight cases of sarcoma of the main pulmonary artery have been published since. In this paper we summarize them and describe a new case of our own, in an endeavour to form a composite clinical and pathological picture by which the disease may be recognized in life.

Froboese's (1928) patient was a woman of 64 who died in heart failure four weeks after admission to hospital. She had had symptoms of cardiac insufficiency for two years. At necropsy the pulmonary artery and its main branches were filled by what appeared to be an embolism. This was removed easily, bringing the intima away from the media ; on microscopy it was found to be a spindle cell sarcoma replacing the intima and invading the media. In the same year Eschbach (1928) described a similar case in a man of 58. The growth appeared to have arisen from the pulmonary conus, and had grown along the intima of the artery, but had not invaded the media. Kudlich and Schuh (1934) gave no clinical details of their patient, a man of 27 , apart from the statement that he had tumours of the lung and mediastinum. At necropsy, tumour masses were found in both lungs and within and around the pulmonary artery. The pulmonary valves were invaded. The sarcoma contained many multinucleated giant cells and spread in the intima of the artery and in the endocardium, also involving the media and adventitia of the artery and neighbouring lymph nodes. Goedel's (1935) patient, a man of 47 , died of heart failure after a few weeks' illness. The pathological findings resembled those of Kudlich and Schuh.

Martin, Tuohy, and Will (1939) described the case of a woman of 46 who died in heart failure four months after the onset of symptoms. The tumour, a polymorphic cell sarcoma, involved the

*Present address: Bernhard Baron Memorial Research Laboratories, Queen Charlotte's Maternity Hospital, London, W.6. entire wall of the pulmonary artery and its main branches, the pulmonary valves, and the endocardium of the right ventricle. Durgin and Ingleby (1946) had a woman aged 53 who died of syncope 12 weeks after the onset of dyspnoea. There appear to have been no abnormal physical signs. At necropsy a mass was found similar to that seen in Froboese's case; it was a "mixed cell" sarcoma. The cause of the terminal syncope was obstruction of the remaining lumen of the artery by blood clot. Moegen (1951) described a man aged 70 who died of heart failure four weeks after the onset of symptoms. At necropsy several sarcomatous masses were present in the pulmonary artery and in the lungs. The pulmonary outlet of the heart was blocked by a blood clot.

The case of Haythorn, Ray, and Wolff (1941) differs from the others in that their patient, a 51-year-old woman, had a long history; because of the radiological appearance of a dense mass in the left lung with large hilar glands, she was given deep $x$-ray therapy. At necropsy there were myxomatous polyps obstructing the artery, and sessile nodules of growth. Secondary deposits were present in the arteries of both lungs, the bronchi, and the lung parenchyma. The tumour was a fibromyxosarcoma.

\section{CAse History}

A man, aged 61 , by occupation a car cleaner, was first seen on February 12, 1958, complaining of difficulty in breathing for six weeks and swelling of the ankles for four weeks. Eight weeks previously he had noticed precordial pain on exertion, accompanied by breathlessness and pain in the left flank, which increased and made him stop work. He had a slight cough. Six weeks previously the breathlessness became worse especially at night, when it came on suddenly and was accompanied by grinding noises in the chest, related to breathing. The breathlessness recurred each night. The cough became productive of yellow sputum. Four weeks previously he passed blood in the urine on one occasion. Three weeks previously breathlessness became much worse and 
was constantly present, even when he sat up in bed. A few days later the right ankle, then the left ankle, became swollen. The pain in the left flank continued and was aggravated by movement.

Previous Illnesses.- - He had been seen at Edmonton Chest Clinic in August, 1955, suffering from acute bronchitis with cough, sputum, breathlessness and sweating at night. He was slightly cyanosed; movement of the chest was poor; rales were heard at the base of the left lung; heart sounds were normal; pulse $84 / \mathrm{min}$. and regular; blood pressure $120 / 80 \mathrm{~mm}$. $\mathrm{Hg}$. Radiography showed an opacity in the posterior basal segment of the left lung. He was treated with a sulphonamide; a fortnight later his cough had improved but he still had yellow sputum. Radiography showed the consolidation to be resolving. He was then given four injections of penicillin, and after a further month he had very little cough or sputum and the pneumonia had almost resolved. In November, 1955, he was discharged from supervision after a normal chest radiograph. He remained fit until the onset of the present illness.

Physical Examination.-He was a well-nourished man, with deep cyanosis, cold hands and feet, and slight finger clubbing.

The pulse was regular (rate $88 / \mathrm{min}$.) and weak in both arms and legs. The blood pressure was 125 / $100 \mathrm{~mm}$. $\mathrm{Hg}$, with inspiratory fading. There was oedema of the lower extremities and over the sacrum. The jugular venous pressure was $9 \mathrm{~cm}$. with wellmarked $a$ wave. The liver was moderately enlarged. The heart was grossly enlarged; a systolic impulse was felt all over the praecordium. On auscultation of the heart, there was a protodiastolic triple rhythm, a widely split second sound. and a soft pulmonary diastolic murmur.

The lungs showed dullness and crepitant rales at the left base.

The muscles of the left calf were tender, and painful on dorsiflexion of the ankle.

Examination of other systems showed no abnormality.

INVESTIGATIONS.-A chest radiograph (Fig. 1) showed gross enlargement of the heart with a rounded left border and enlargement of the pulmonary conus, hiding the left pulmonary artery. In the right lung, the vascular markings were much reduced but regular, and the pulmonary artery was very small. The upper part of the left lung was normal, but a diffuse shadow obscured the left base.

An electrocardiogram showed sinus rhythm at a rate of $88 / \mathrm{min}$.; right axis deviation and partial right bundle branch block; and well-marked SI, inverted $T$ in II and III, AVR, AVF, $V_{1}, V_{2}$, and $V_{3}$. These changes were regarded as consistent with pulmonary embolism.

A blood count showed haemoglobin 105\%; leucocytes 19,800 per c.mm. (polymorphs $64 \%$, small lymphocytes $22 \%$, large lymphocytes $8 \%$, monocytes $6 \%$ ).

Urine.-No abnormal constituents were found.
Sputum.-No pathogenic bacteria or malignan cells were found.

Blood urea was $39 \mathrm{mg} . / 100 \mathrm{ml}$; blood culture sterile; and plasma proteins within normal limits.

Clinical Diagnosis. - This was pulmonaryen thrombo-embolism, consequent on thrombo-phlebitisof the legs; possibly a sequel to coronary thrombosis: with a consequent renal embolism.

TrEATMENT.--Treatment consisted of oxygen, anticoagulants, and subsequently digitalization. A very $\overrightarrow{\vec{x}}$ satisfactory diuresis was obtained with chlorothiazide? but some oedema of the ankles persisted and the $A$ jugular venous pressure remained raised. Although the patient felt more comfortable in the oxygen tentw he remained deeply cyanosed.

Progress.-On February 28 oedema had decreased and the jugular venous pressure was about $4 \mathrm{~cm} . \vec{\square}$ above the sternal angle with a large $a$ wave. The pulmonary diastolic murmur had disappeared. Signs尺్ of collapse of the left lower lobe persisted. The sputum was purulent. Staphylococcus aureus wasष grown on culture.

Radiography showed a slight decrease in the size $\vec{\theta}$ of the heart; a small infiltration was present in the upper zone of the right lung.

Four days later his condition was deteriorating. temperature $98.8^{\circ} \mathrm{F}$., pulse $100 / \mathrm{min}$. The large wave had been replaced by a $v$ wave and a tricuspid $\overline{0}$ systolic murmur was present; splitting of the second? sound persisted. Radiography showed a small abscess $\stackrel{\mathbb{Q}}{2}$ in the right upper zone. Treatment was started with $\overrightarrow{\vec{F}}$ tetracycline, but the sputum increased and hecame응 steaciily more purulent.

On March 31 (Figs. 2 and 3) radiography showed that the right upper lobe had disintegrated into abscess. cavities. The vascular markings in the left lung had $\overrightarrow{5}$ become less prominent and a dense comma-shaped shadow was seen behind the pulmonary conus in the $\frac{x}{x}$ position of the left pulmonary artery. This was taken to be the shadow cast by an embolism in the pulmonary artery.

The patient improved again and his temperature $\frac{0}{3}$ fell to normal, but he produced a small amount of 0 purulent sputum infected with staphylococci until death, in spite of treatment with antibiotics. He had $\frac{D}{2}$ slight pain in the left side of the chest and the sputum was blood-stained on May 29.

In July, because it was thought that deterioration in his condition might be due to prolonged treatment $N$ with chlorothiazide, the plasma electrolytes were estimated, but found to be within normal limits.

On August 5 radiography showed that the heart was smaller. The abscess cavities in the right upper lobe were represented by a few thin-walled cysts, the $\mathbb{D}$ left lung had become almost avascular, and collapse of the left lower lobe persisted. The shadow thought to be a pulmonary embolism was still present in the left hilum (Fig. 4).

On August 11 the right arm became useless, and the patient became disorientated and complained of headache. This was attributed to a cerebral embolism. 
After a gradual improvement, lasting six days, the arm again became totally paralysed as did the right leg on the following day. There was loss of sensation and movement of the entire right side, apart from the face. The tendon reflexes were brisk; ankle clonus was present, and both plantar responses were flexor. Retinoscopy showed no abnormality. The pulse was regular, rate $\cdot 80 / \mathrm{min}$., blood pressure 160 / $120 \mathrm{~mm}$. $\mathrm{Hg}$, deep cyanosis, and jugular venous pressure $4 \mathrm{~cm}$. with a large $v$ wave. The heart was clinically slightly smaller than it had been, but there was a powerful precordial thrust. Summation gallop was present with wide splitting of the second sound. The latter disappeared by August 27. The patient gradually sank into coma and died on October 10 , 1958.

\section{NECROPSY}

External Appearances.-The body was that of a poorly nourished middle-aged man, with no sacral or leg oedema.

The larynx, trachea, and bronchi were slightly inflamed and contained a small amount of mucopus. The small bronchi were thickened and moderately dilated.

The lungs showed suppurative bronchopneumonia in all areas, affecting particularly the left lower lobe. In the right upper lobe there was an abscess $4 \mathrm{~cm}$. in diameter, the wall of which consisted of soft, dark, necrotic material.

The pulmonary artery contained a cream-coloured, firm, elastic mass in which were small, elongated, haemorrhagic streaks. The mass was adherent to one cusp of the pulmonary valve, which was competent. A similar mass, $3 \mathrm{~cm}$. in diameter and well localized, lay beneath the bifurcation of the pulmonary artery, and at this point was directly continuous with the mass in the artery, where all the coats had been invaded and replaced by the growth. The left superior pulmonary vein was involved in this tumour and had been invaded by it. Distal extension into the branches of the pulmonary artery had involved the secondary divisions. There were ante-mortem thrombi distal to the tumour, and in addition several cream-coloured nodules were present in the walls of the small branches of the pulmonary artery (Fig. 5).

The pleural sac was obliterated by firm fibrous adhesions over the right upper lobe.

The mediastinal lymph nodes appeared large and oedematous with no naked-eye evidence of invasion by tumour.

The heart weighed $450 \mathrm{~g}$. and showed considerable hypertrophy and dilatation of both the right ventricle and right atrium. The tricuspid valve admitted six fingers. The mitral valve was normal. Neither the myocardium nor the endocardium, except for the pulmonary valve, had been involved by growth (Fig. 6). The coronary arteries were normal. The pericardial sac contained $200 \mathrm{ml}$. of straw-coloured fluid.

The left and right posterior tibial veins contained organized thrombus.
A large infarct occupied the occipital and parietal lobes of the left cerebral hemisphere (Fig. 7). In the middle of this, about $\frac{1}{2} \mathrm{~cm}$. beneath the surface of the brain, was a firm, cream-coloured, haemorrhagic tumour about $3 \mathrm{~cm}$. in diameter. The cerebral vessels showed moderate atheroma involving the basilar and middle cerebral arteries in particular. The meninges showed slight oedema only.

The alimentary system and endocrine glands exhibited no abnormality.

There was an old infarct in the left kidney. The ureters, bladder, and prostate were normal.

The spleen was normal.

Histology.-The main pulmonary artery showed fibrosarcoma tissue in which the predominant cell was spindle shaped with acidophil cytoplasm (Fig. 8). Most of the nuclei of these cells were fairly well condensed, but some were vesicular. The tumour showed moderate pleomorphism but was not rich in mitoses. Much intercellular connective tissue was present. Covering this small tumour mass, and lying nearer the lumen of the vessel, was organized thrombus containing large acellular areas.

A smaller branch of the right pulmonary artery showed a more cellular tumour, but on the whole was similar to the one described above. There was only a thin layer of organized thrombus overlying the tumour at this site.

The pulmonary valve showed mainly organized thrombus, with only scattered islands of fibrosarcoma cells furthest away from the lumen of the valve.

A mediastinal lymph node showed invasion by fibrosarcoma cells.

The brain tumour showed a cellular fibrosarcoma, the individual cells of which were pleomorphic, but mainly spindle shaped (Fig. 9). Many of the cells were large and contained round vesicular nuclei with prominent nucleoli.

\section{Discussion}

Clinical Features and Diagnosis.-Sarcoma of the pulmonary artery has been described in adults of both sexes. The patients have suffered from breathlessness, precordial pain and syncope, or epigastric pain. They have subsequently developed enlargement of the heart and the signs of right heart failure with oedema and anasarca.

In most cases a generalized systolic murmur has been present. It was attributed by Moegen (1951) and Froboese (1928) to the mitral valve, by Eschbach (1928) to the aortic valve. Eschbach quoted a case of sarcoma of the pulmonary valve, described by Mandelstamm (1923), who thought that the systolic murmur was aortic in origin. Haythorn and others (1941) found no murmur, and the patient described by Durgin and Ingleby (1946) was told that she had nothing wrong with her heart 10 days before death. Martin and others (1939) found tricuspid murmur. 


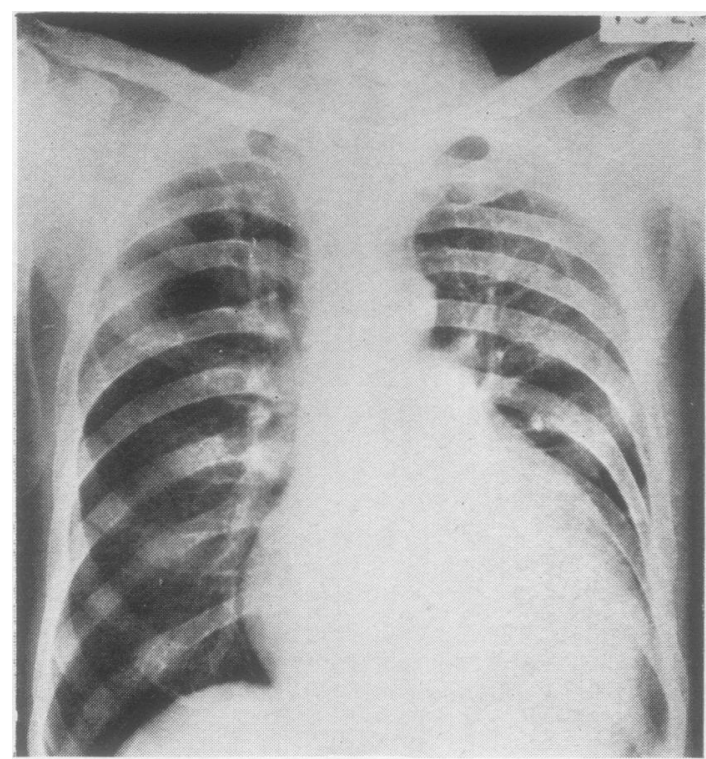

Fio. 1

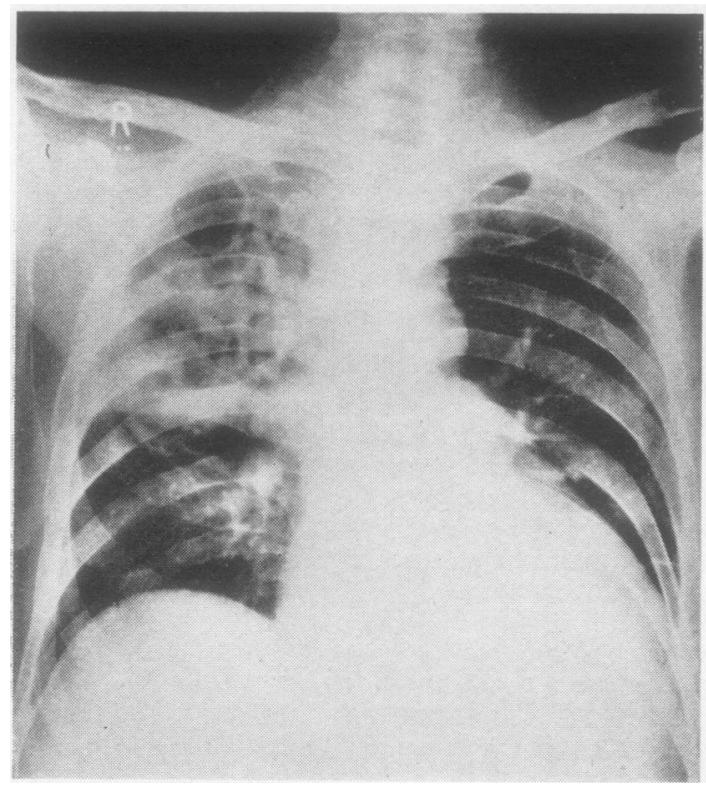

Fig. 2

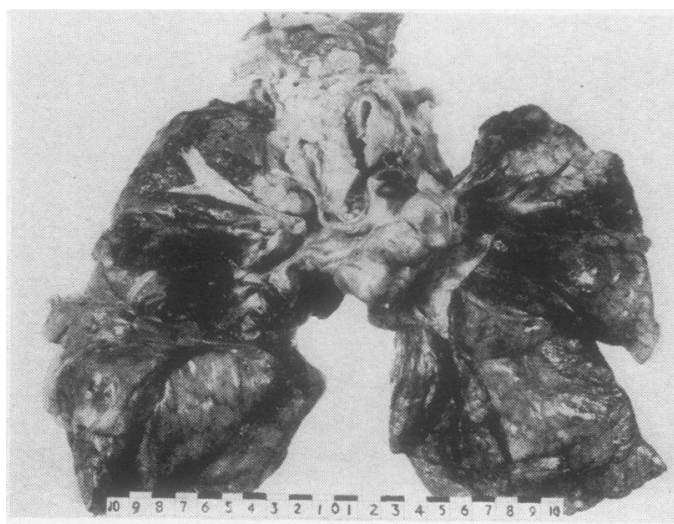

Fic. 5

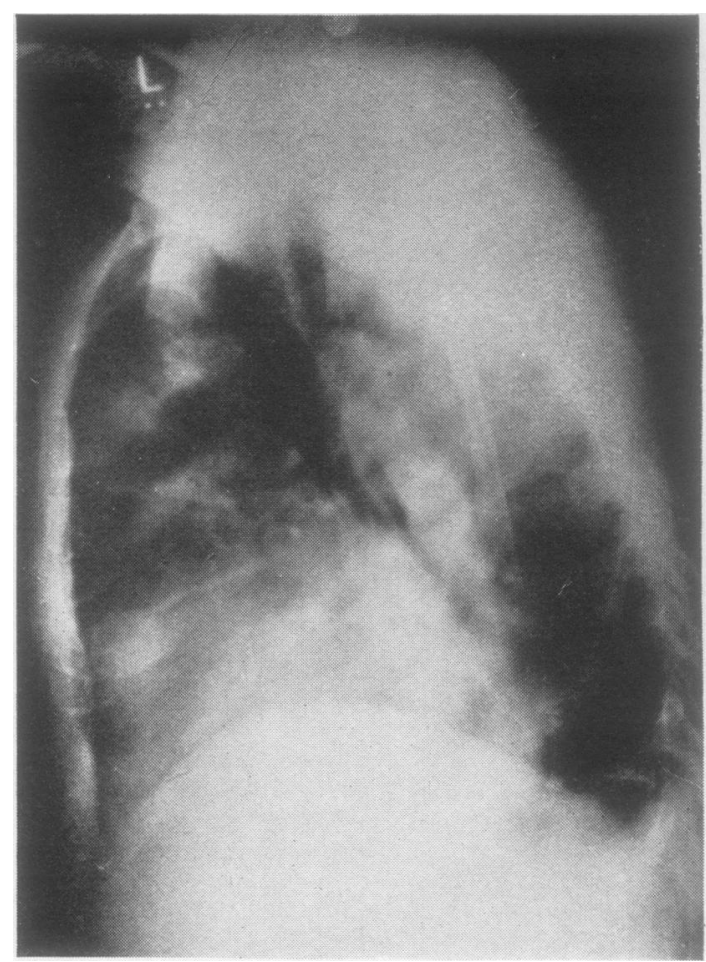

Fig. 3

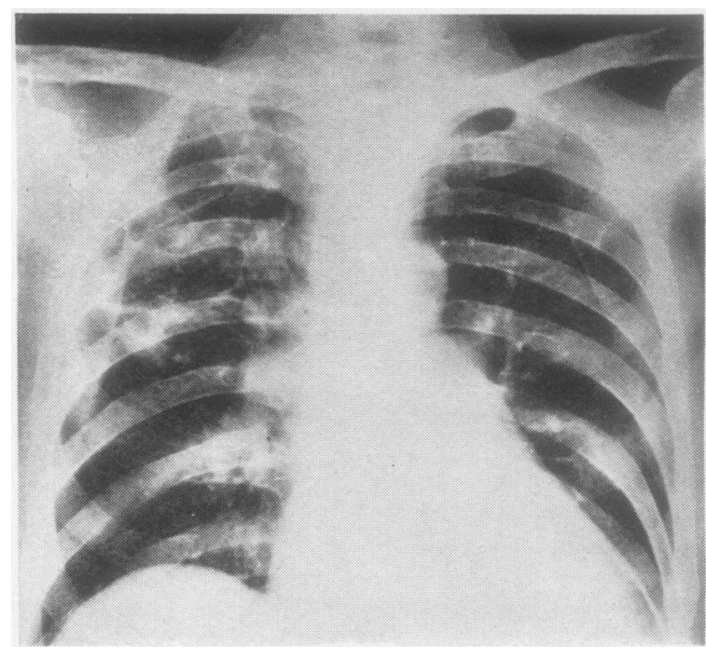

Fig. 4

FIG. 1.-Chest radiograph, February 12, 1958.

FIG. 2.-Chest radiograph, February 28, 1958.

Fig. 3.-The same, lateral view. The mass in the position of the left pulmonary artery is seen extending from the knuckle of the aorta downwards and to the left.

FIG. 4.-Chest radiograph, August 5, 1958.

FIG. 5.-Partially dissected specimen showing, in addition to the main tumour mass, a smaller extension of the growth in a branch of the right division of the pulmonary artery. 


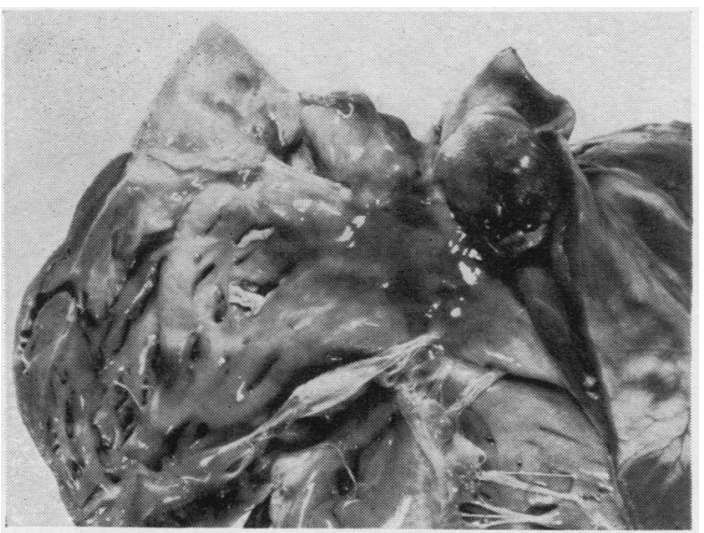

Fig. 6.-The tumour involving the pulmonary valve. Adjacent to this is a pedunculated, partially organized, thrombus.

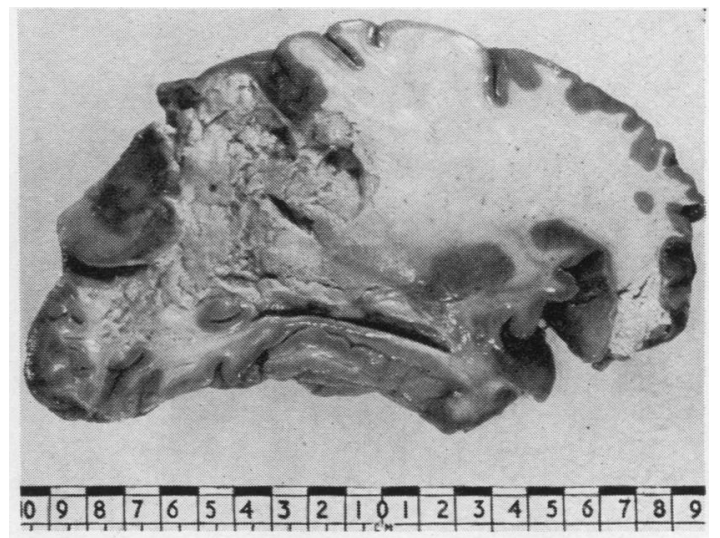

FIG. 7.-Left cerebral hemisphere showing a secondary sarcomatous deposit surrounded by an area of infarction.

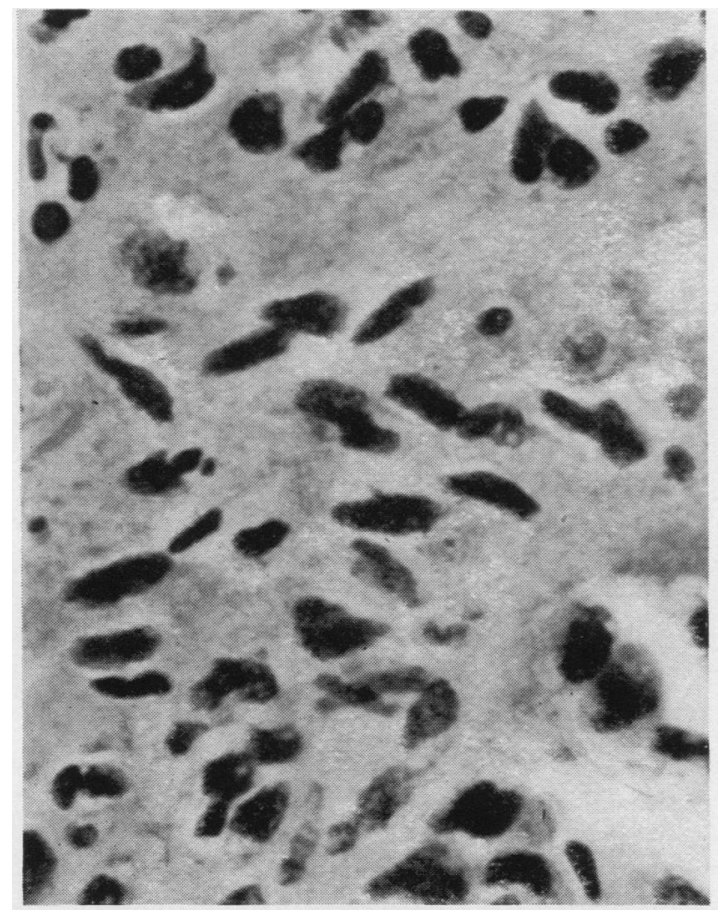

FIG. 8. Section of primary tumour showing pleomorphism and spindle-shaped fibroblastic cells. Haematoxylin and eosin,
$\times 500$. 
In our case the systolic murmur was not present at first, and was undoubtedly of tricuspid origin. There was also a pulmonary diastolic murmur initially, which we suppose was due to pulmonary hypertension, although it disappeared later. The split second sound was identified independently by several observers. It was followed at first by pulmonary diastolic murmur and protodiastolic gallop. Subsequently it was heard with summation gallop. The disappearance of the pulmonary element of the second sound late in the disease may have been due to invasion of the valves. This change could have occurred for other reasons and so has no diagnostic significance.

The rise in blood pressure in the late stages of the disease appears to have been due to anoxia, for the patient had no hypertension in 1955. Such a rise has not been observed in other published cases.

Previous authors, like ourselves, have attributed heart failure in their cases to one of the common causes of right heart failure, favouring pulmonary embolism. This is the most likely cause of sudden right heart failure due to occlusion of the pulmonary arteries in a previously healthy person. The original picture in our case corresponded closely to this, but on repeated questioning both our patient and his wife denied that there had been a sudden incident after which he felt himself to be very much worse. The same lack of sudden incident is seen in the other case histories. although the patient described by Durgin and Ingleby (1946) died of syncope.

That our patient was not suffering from thrombosis of the pulmonary artery was suggested by the absence of antecedent pulmonary hypertension, pulmonary stenosis, or leukaemia, and the fact that the main branches of the pulmonary artery (apart from that to the left lower lobe) were decreased in size. In fact, in our patient thrombi were found attached to the walls of small branches peripheral to the tumour, and at necropsy in other cases besides that of Durgin and Ingleby (1946) thromboses have been found.

Similar intractable heart failure may be seen in cases of malignant tumour of the right heart, but tricuspid obstruction, caval obstruction, and clinically significant pericarditis are common. Prichard (1951), in his Case 2, found pericardial friction and a very high venous pressure, and at necropsy the right ventricle and pulmonary artery and its branches were almost filled by myxomatous tissue arising from the tricuspid valve. Neither caval obstruction nor clinically significant pericarditis have been reported in cases of pulmonary artery sarcoma, although the pe $\overrightarrow{\dot{\varphi}}-$ cardium was involved by the tumour in Goede (1935) case.

Our patient's electrocardiogram had diagnostic significance. It was similar to theit described by Moegen (1951) and showed evidence of right heart strain. The patient of Martm and others (1939) had a low-voltage curve a $\overrightarrow{\text { tel }}$ isoelectric TI.

Secondary deposits in the lungs and mediat tinum have been found radiologically or ${ }_{a}$ necropsy by several authors. They may arise by direct extension of the growth. Our patient haf no involvement of the lung beyond the vesse but a pulmonary vein had been invaded by the growth and there was a secondary deposit in the brain. Only Kudlich and Schuh (1934) previousty have found a secondary deposit outside the chent -in the suprarenal glands. In neither case di the metastasis help in diagnosis.

The radiological changes that we observed have not been described previously. Other authos have described tumours and infarction of the lungs and mitralization of the heart. We found progressive devascularization of our patiens lungs, which had been normal two years befofe his final illness. At first the right pulmonamy artery was small and the peripheral vasculpr markings were reduced, then a dense shadow which we took to be embolism, appeared in the position of the left pulmonary artery. Subse. quently an abscess developed in the right uppo lobe and finally the vascular markings in the lest upper lobe became attenuated.

Progressive devascularization with narrowiag of main branches of the pulmonary artery may be diagnostic, although a variety of spaceoccupying lesions of the mediastinum can compregs the pulmonary artery causing stenosis. Winter (1958) described a case of an anterior mediastinal thymoma which pressed on the pulmonary value giving rise to a systolic murmur, a steno pressure gradient, and evidence of right heat strain; the published radiographs and angiơ cardiogram show no devascularization of the lungs. One of us was recently shown a case th Mr. Michael Bates (1959), in which attenuation of the vascular shadows was noticed in the left lurtig. A woman aged 57 complained of dyspnoea art dysphagia for three months. There was clinic\&l evidence of a mediastinal tumour. A faint systolic murmur was present at the base of the heart, but there was no evidence of heart failure. A large anterior mediastinal mass and loss क्षे vascular markings in the left lung were seen $\mathrm{P}$ the chest radiograph. On endoscopy the trach 
was flattened, the left main bronchus was stenosed, and the oesophagus compressed. At operation the mass was found to be a secondary deposit from a carsinoma of the left upper lobe bronchus, compressing the left main branch of the pulmonary artery, which was dilated in front of the stenosis.

In most cases death has followed rapidly after the development of heart failure, but the cases reported by Froboese (1928) and by Haythorn and others (1941) ran a prolonged course after heart failure developed. Our patient was kept alive for some months by intensive treatment.

The quintessence of the disease is steadily progressive right heart failure with narrowing of the main branches of the pulmonary artery, and possibly tumours in the mediastinum and lungs, but without the catastrophic features of pulmonary embolism or coronary thrombosis, or evidence of preceding pulmonary hypertension.

OrIGIN OF THE GROWTH.-We agree with previous authors that these tumours arise from the tissues of the pulmonary artery. However, certain other possibilities deserve mention.

Intrathoracic sarcomas may be classified as follows :

(a) Primary tumours.

(i) Arising in mediastinal structures (including great vessels).

(ii) Arising in tissues in and around the vertebral column.

(iii) Arising in the lung.

(b) Secondary tumours.

Almost all sarcomas of the lung are secondary tumours, but they may differ in histological appearance from the primary growth. For example, metastatic osteogenic sarcomas may contain no bone tissue and metastatic melanosarcomas (if they may be called sarcomas) may contain no pigment. By the time pulmonary metastases are obvious, widespread systemic dissemination of the tumour has usually occurred. The possibility of the tumour described in this paper arising as part of a thrombo-embolism of the pulmonary artery, with little other metastatic spread, seems extremely unlikely ; in recent years only one case of obstruction of the main pulmonary artery by tumour embolism has been recorded (Laurain, 1957), and in this case the primary tumour was obvious.

Primary sarcoma of the bronchus as described by Lewis (1947) is extremely rare and many cases reported in the early literature would now be recognized as undifferentiated small-cell bronchogenic carcinomas. Tumours arising outside the great vessels do not normally invade the media, although they may injure the vasa vasorum to cause necrosis of the wall with rupture of the vessel. We found no histological evidence of neurofibrosarcoma and the tumour did not arise in the paravertebral region. Thus the only possible sites of origin of this tumour are the tissues of the pulmonary artery itself and the pulmonary valve. We have found only one sarcoma of the pulmonary valve recorded (by Mandelstamm, 1923), apart from some of the tumours considered here which involved the valve and may possibly have originated there.

In our case special staining failed to reveal any muscle striations, but a tumour of the heart is not excluded on this count, for fibrosarcoma of the heart is well known, and Stout (1946) believes that rhabdomyoblastic cells can form collagen and closely resemble fibroblasts in appearance. However, macroscopic and microszopic examination of the heart and pulmonary artery of our patient strongly suggested that the sarcoma arose from the wall of the pulmonary artery in the region of its bifurcation, and was only attached secondarily to the heart by the pulmonary valve.

The general pattern of growth of these tumours is invasion of the intima of the pulmonary arteries in continuity, with only localized penetrations of the media and surrounding structures. Distant metastases are rare, but invasion of local lymph nodes is common.

No special aetiological factors can be discerned in the cases so far described.

Treatment.-There seems to be no possibility of successful treatment of this type of growth unless the diagnosis is made at a much earlier stage than that at which patients have presented up to now. Only Haythorn and others (1941) attempted treatment, in ignorance of the nature of the tumour that they were irradiating, and without success.

\section{SUMmaRY}

A case of primary sarcoma of the main pulmonary artery is described, and eight previously recorded cases in which primary sarcomata have arisen in the intima, media, or adventitia of the main pulmonary artery are reviewed.

Primary sarcoma of the main pulmonary artery causes unremitting, progressive, right-sided heart failure, with evidence of obstruction of the pulmonary artery or stenosis of the valves.

Extension of the growth may be seen radiologically in the mediastinum or in the lungs. 
Progressive devascularization of the lungs may be the most important diagnostic feature of the disease.

Extrathoracic secondary deposits are exceptional.

Correct diagnosis may possibly be made during life, but at the stage of the disease at which patients have presented up to now, no useful treatment can be foreseen.

We wish to express our gratitude to Dr. $\mathrm{H}$. Loewenthal, of Chase Farm Hospital, for translations ; to Dr. J. Prendiville, of the same hospital, and Dr. J. F. Heggie, of the North Middlesex Hospital, for the use of pathological material; to Mr. M. Bates, of the North Middlesex Hospital, for permission to use his case history; to Dr. J. H. Pratt-Johnson, of
Tottenham Chest Clinic, for valuable advice and suggestions ; and to Mrs. B. Glass, of Edmonton Ches Clinic, for secretarial assistance.

\section{REFERENCES}

Bates, M. (1959). Personal communication.

Durgin, B., and Ingleby, H. (1946). Clinics, 5, 182.

Eschbach, H. (1928). Beitr. path. Anat., 80, 672.

Goedel, A. (1935). Frankfurt. Z. Path., 49, 1.

Haythorn, S. R., Ray, W. B., and Wolff, R. A. (1941). Amer. $\vec{A}$

Path., 17, 261.

ores, L. (1924). In Handbuch der speziellen pathologischen Anatorfie und Histologie, ed. F. Henlie and $O$. Lubarsch, Vol. 2, pp. 608 786. Springer, Berlin.

Kudlich, H., and Schuh, W. (1934). Virchows Arch. path. Anat., 294, 113.

Laurain, A. R. (1957). Amer. J. clin. Path., 27, 664.

Lewis, Ivor (1947). Proc. roy. Soc. Med., 40, 19

Mandelstamm, M. (1923). Virchows Arch. path. Anat., 245, 43. Martin, W. C., Tuohy, E. L., and Will, C. (1939). Amer. Heart $17,728$.

Moegen, P. (1951). Z. Kreisl.-Forsch., 40, 150.

Prichard, R. W. (1951). A.M.A. Arch. Path., 51, 98.

Stout, A. P. (1946). Ann. Surg., 123, 447.

Winter, B. (1958). Amer. Heart J., 55, 18 